\title{
Répartition modulo 1 dans un corps de séries formelles sur un corps fini
}

\author{
par \\ Mireille Car (Marseille)
}

Introduction. Soit $q$ une puissance d'un nombre premier $p$ et soit $\mathbb{F}_{q}$ le corps fini à $q$ éléments. Une certaine analogie entre l'arithmétique de l'anneau $\mathbb{Z}$ des entiers rationnels et celle de l'anneau $\mathbb{F}_{q}[T]$ a conduit à étendre à $\mathbb{F}_{q}[T]$ de nombreuses questions de l'arithmétique classique. L'équirépartition modulo 1 est une de ces questions. Le corps des nombres réels est alors remplacé par le corps $\mathbb{F}_{q}\left(\left(T^{-1}\right)\right)$ des séries de Laurent formelles, complété du corps $\mathbb{F}_{q}(T)$ des fractions rationnelles pour la valuation à l'infini et l'intervalle [0,1[ est remplacé par l'idéal de valuation. L. Carlitz [1] a donné une définition de l'équirépartition modulo 1 dans le corps $\mathbb{F}_{q}\left(\left(T^{-1}\right)\right)$ qui s'est révélée fructueuse puisqu'elle permet l'utilisation d'un critère de Weyl [1], [7], la généralisation des premiers résultats de Weyl [2], [3], du théorème de Koksma [7], ou du théorème de Vinogradov [8]. Il est bien connu que la suite $(\sqrt{n})$ est équirépartie modulo 1 . Il est donc naturel de poser la question de l'équirépartition modulo 1 de la suite $\left(H^{1 / 2}\right), H$ décrivant la suite des polynômes de $\mathbb{F}_{q}[T]$ admettant une racine carrée $H^{1 / 2}$ dans le corps $\mathbb{F}_{q}\left(\left(T^{-1}\right)\right)$, et, plus généralement, celle de la suite $\left(H^{1 / l}\right), H$ décrivant la suite des polynômes de $\mathbb{F}_{q}[T]$ admettant une racine $l$-ième $H^{1 / l}$ dans le corps $\mathbb{F}_{q}\left(\left(T^{-1}\right)\right)$. C'est ce qui est fait dans ce qui suit, où l'on précise ce que l'on entend par racine $l$-ième. On démontre que pour $l \geq 2$, la suite $\left(H^{1 / l}\right)$ est équirépartie modulo 1 , et que pour $l \geq 3$, la suite $\left(P^{1 / l}\right)$ est équirépartie modulo $1, P$ décrivant la suite des polynômes irréductibles de $\mathbb{F}_{q}[T]$ admettant une racine $l$-ième dans le corps $\mathbb{F}_{q}\left(\left(T^{-1}\right)\right)$.

\section{Préliminaires}

I.1. Notations et rappels. On pose $\mathbf{R}=\mathbb{F}_{q}[T], \mathbf{K}=\mathbb{F}_{q}(T)$. La valuation à l'infini sur le corps $\mathbf{K}$ est l'application $\nu$ de $\mathbf{K}$ dans $\mathbb{Z} \cup\{\infty\}$ définie par $\nu(0)=\infty$ et $\nu(A / B)=\operatorname{deg} B-\operatorname{deg} A$, si $A$ et $B$ sont des polynômes non nuls.

Le complété de $\mathbf{K}$ pour la valuation $\nu$ est le corps $\mathbf{K}_{\infty}=\mathbb{F}_{q}\left(\left(T^{-1}\right)\right)$ des séries de Laurent formelles 


$$
y=\sum_{n=-\infty}^{\infty} y_{n} T^{n}
$$

où $y_{n} \in \mathbb{F}_{q}$, les coefficients $y_{n}$ étant tous nuls pour $n$ assez grand. On prolonge la valuation $\nu$ à $\mathbf{K}_{\infty}$ en posant pour $y \neq 0$,

$$
\nu(y)=-\sup \left\{n \in \mathbb{Z} \mid y_{n} \neq 0\right\} .
$$

Soit $\mathfrak{P}$ l'idéal de valuation de $\mathbf{K}_{\infty}$. Le corps $\mathbf{K}_{\infty}$ est localement compact, un système fondamental de voisinages compacts de l'origine étant fourni par les puissances de l'idéal $\mathfrak{P}$. A constante multiplicative près, il existe une seule mesure de Haar sur $\mathbf{K}_{\infty}$. On choisit la mesure de Haar $\mu=d t$ normalisée à 1 sur l'idéal de valuation, c'est-à-dire telle que

$$
\int_{\mathfrak{P}} d t=1 \text {. }
$$

Tout $y \in \mathbf{K}_{\infty}$ s'écrit de façon unique comme somme

$$
y=[y]+\{y\}, \quad[y] \in \mathbf{R}, y \in \mathfrak{P} .
$$

Si $y \in \mathbf{K}_{\infty}$ s'écrit $y=\sum_{n=-\infty}^{\infty} y_{n} T^{n}$, on pose

$$
\operatorname{Res}(y)=y_{-1} \text {, }
$$

si de plus $y \neq 0$, on pose

$$
\operatorname{sgn}(y)=y_{-\nu(y)} .
$$

Soit $\psi: \mathbb{F}_{q} \mapsto \mathbb{C}$ un caractère additif non trivial. Pour tout $y \in \mathbf{K}_{\infty}$, on pose

$$
E(y)=\psi(\operatorname{Res}(y)) .
$$

Alors $E$ est un caractère additif non trivial de $\mathbf{K}_{\infty}$.

Pour tout $a \in \mathbf{K}_{\infty}$ et tout $r \in \mathbb{Z}$, soit

$$
\mathcal{B}(a, r)=\left\{y \in \mathbf{K}_{\infty} \mid \nu(y-a)>r\right\} .
$$

Alors, on a (cf. [4])

$$
\mu(\mathcal{B}(a, r))=q^{-r} .
$$

On notera $\mathbb{F}_{q}^{*}, \mathbf{R}^{*}, \mathbf{K}^{*}, \mathbf{K}_{\infty}^{*}$ l'ensemble des éléments non nuls de $\mathbb{F}_{q}, \mathbf{R}, \mathbf{K}$, $\mathbf{K}_{\infty}$ respectivement.

I.2. Équirépartition modulo 1. Soit $\Theta=\left(\theta_{n}\right)$, une suite à valeurs dans $\mathbf{K}_{\infty}$. Pour tout entier $N>0$, tout $b \in \mathfrak{P}$ et tout $r \in \mathbb{Z}$, soit $A(\Theta, N, b, r)$ le nombre d'entiers $n \in\{1, \ldots, N\}$ tels que $\left\{\theta_{n}\right\} \in \mathcal{B}(b, r)$. Soit alors,

$$
\Delta(\Theta, N, b, r)=\frac{A(\Theta, N, b, r)}{N}-\mu(\mathcal{B}(a, r))=\frac{A(\Theta, N, b, r)}{N}-q^{-r},
$$

et

$$
D(\Theta, N)=\sup _{b \in \mathfrak{P}, r \in \mathbb{Z}}|\Delta(\Theta, N, b, r)|
$$


Définition I.2.1 (Carlitz). Une suite $\Theta$ à valeurs dans $\mathbf{K}_{\infty}$ est dite équirépartie modulo 1 si la suite $(D(\Theta, N))$ tend vers 0 lorsque $N$ tend vers $\infty$.

On a alors le critère de Weyl (cf. [1], [7]) :

ThÉORÈme I.2.2. Une suite $\Theta$ à valeurs dans $\mathbf{K}_{\infty}$ est équirépartie modulo 1 si et seulement si pour tout $H \in \mathbf{R}^{*}$ non nul, la suite $\left((1 / N) \sum_{n=1}^{N} E\left(H \theta_{n}\right)\right)$ tend vers 0 lorsque $N$ tend vers $\infty$.

I.3. Les racines $l$-ièmes dans $\mathbf{K}_{\infty}^{*}$. Soit un entier $l \geq 2$ non divisible par la caractéristique $p$ du corps $\mathbb{F}_{q}$. Dans $\mathbb{F}_{q}^{*}$ il y a exactement

$$
r=\frac{q-1}{\text { p.g.c.d. }(l, q-1)}
$$

puissances $l$-ièmes. Soit $\mathcal{L}$ l'ensemble de ces $r$ puissances $l$-ièmes.

LEMME I.3.1. Soient

$$
a=\sum_{j=-\infty}^{N} a_{j} T^{j}, \quad b=\sum_{j=-\infty}^{n} b_{j} T^{j}
$$

des éléments de $\mathbf{K}_{\infty}^{*}$. Alors $a=b^{l}$ si et seulement si les trois conditions suivantes sont réalisées:

(i) $N=\ln$,

(ii) $a_{N}=b_{n}^{l}$,

(iii) pour tout entier $k \geq 1$, on a

$$
a_{N-k}=l b_{n}^{l-1} b_{n-k}+\sum_{\substack{i_{1}+\ldots+i_{l}=N-k \\ n-k<i_{1} \leq n \\ n-k<i<\\ n-k<i_{l} \leq n}} b_{i_{1}} \ldots b_{i_{l}} .
$$

Dém on stration. Immédiate.

Proposition I.3.2. Soit $a \in \mathbf{K}_{\infty}^{*}$. Alors, il existe $b \in \mathbf{K}_{\infty}^{*}$ tel que $a=b^{l}$ si et seulement si $\nu(a) \equiv 0 \bmod l$ et $\operatorname{sgn}(a) \in \mathcal{L}$. Dans ce cas il y a exactement $r$ éléments $b \in \mathbf{K}_{\infty}^{*}$ tels que $a=b^{l}$.

Démonstration. Les conditions (i) et (ii) du lemme ci-dessus montrent la nécessité des conditions. Soit $a=\sum_{j=-\infty}^{l n} a_{j} T^{j}$ un élément de $\mathbf{K}_{\infty}^{*}$ tel que $\nu(a)=-\ln$ et tel que $\operatorname{sgn}(a)=a_{l n}$ soit une puissance $l$-ième dans $\mathbb{F}_{q}$. Soit $\beta \in \mathbb{F}_{q}^{*}$ tel que $\operatorname{sgn}(a)=\beta^{l}$. On pose $b_{n}=\beta$. Ensuite, on détermine $b_{n-1}, b_{n-2}, \ldots, b_{n-k}, \ldots$ à l'aide des relations (I.3.2). On pose $b=\sum_{j=-\infty}^{n} b_{j} T^{j}$. Alors, $a=b^{l}$. De plus, toute solution $\beta$ de l'équation $\operatorname{sgn}(a)=\beta^{l}$ détermine une et une seule solution $b$ de l'équation $a=b^{l}$.

Nous pouvons maintenant définir la fonction racine $l$-ième. 
Soit $\mathcal{L}=\left\{\alpha_{1}, \ldots, \alpha_{r}\right\}$. Pour chaque indice $i \in\{1, \ldots, r\}$, on choisit $\beta_{i} \in \mathbb{F}_{q}^{*}$ tel que $\alpha_{i}=\beta_{i}^{l}$. Si $a \in \mathbf{K}_{\infty}^{*}$ est tel que $\nu(a) \equiv 0 \bmod l$ et $\operatorname{sgn}(a) \in \mathcal{L}$, on désigne par $a^{1 / l}$ l'unique élément de $\mathbf{K}_{\infty}^{*}$ tel que

$$
\left(a^{1 / l}\right)^{l}=a, \quad \operatorname{sgn}\left(a^{1 / l}\right)=\beta_{i} \quad \text { si } \operatorname{sgn}(a)=\alpha_{i} .
$$

I.4. L'ordre sur $\mathbf{R}$. On considère une bijection $i \mapsto \mathcal{X}_{i}$ de $\{0, \ldots, q-1\}$ sur $\mathbb{F}_{q}$ telle que $\mathcal{X}_{0}=0$. On peut aussi supposer, mais ce n'est pas indispensable, que $\mathcal{X}_{1}=1$. On ordonne $\mathbb{F}_{q}$ en posant $\mathcal{X}_{i}<\mathcal{X}_{i+1}$ pour tout $i \in\{0, \ldots, q-1\}$. Soit un entier positif $n$. On écrit $n$ en base $q$ :

$$
n=n_{0}+n_{1} q+\ldots+n_{s} q^{s}, \quad n_{i} \in\{0, \ldots, q-1\},
$$

et on pose

$$
H_{n}=\mathcal{X}_{n_{0}}+\mathcal{X}_{n_{1}} T+\ldots+\mathcal{X}_{n_{s}} T^{s} .
$$

L'application $n \mapsto H_{n}$ est une bijection de $\mathbb{N}$ sur $\mathbf{R}$ telle que

$$
m \leq n \Rightarrow \operatorname{deg} H_{m} \leq \operatorname{deg} H_{n}
$$

On ordonne $\mathbf{R}$ en posant, pour tout entier naturel $n$,

$$
H_{n}<H_{n+1} \text {. }
$$

II. Le théorème. Soit un entier $l \geq 2$ non divisible par la caractéristique $p$ du corps $\mathbb{F}_{q}$. L'ensemble $\mathcal{L}=\left\{\alpha_{1}, \ldots, \alpha_{r}\right\}$ des puissances $l$-ièmes de $\mathbb{F}_{q}^{*}$ introduit au paragraphe précédent est maintenant indexé suivant l'ordre croissant de $\mathbb{F}_{q}$.

Un polynôme $K \in \mathbf{R}^{*}$ admet une racine $l$-ième dans $\mathbf{K}_{\infty}^{*}$ si et seulement si $\operatorname{deg} K \equiv 0 \bmod l$ et $\operatorname{sgn}(K) \in \mathcal{L}$. Soit $\mathbf{L}$ l'ensemble de ces polynômes. Soit $\left(L_{n}\right)$ la suite de ces polynômes, indexée suivant l'ordre croissant de $\mathbf{R}$. Soit $\mathbf{I}$ l'ensemble des polynômes irréductibles de $\mathbb{F}_{q}[T]$ et soit $\left(P_{n}\right)$ la suite formée par les polynômes de $\mathbf{L} \cap \mathbf{I}$, indexée suivant l'ordre croissant de $\mathbf{R}$.

Dans ce qui suit, à l'aide du critère de Weyl, on démontre le théorème suivant.

ThÉORÈme. La suite $\left(L_{n}^{1 / l}\right)$ est équirépartie modulo 1 . De plus, pour $l \geq 3$, la suite $\left(P_{n}^{1 / l}\right)$ est équirépartie modulo 1.

II.1. Le lemme fondamental

LEMme II.1.1 (Lemme préliminaire). Soit $k$ un entier naturel et soit $H \in$ $\mathbf{R}^{*}$. Soit $L \in \mathbf{L}$ de degré lk. Alors, pour tout $Z \in \mathbf{R}$ tel que $\operatorname{deg} Z<$ $(l-1) k-\operatorname{deg} H-1$, on $a$

(i) $(L+Z) \in \mathbf{L}$,

(ii) $\operatorname{Res}\left(H L^{1 / l}\right)=\operatorname{Res}\left(H(L+Z)^{1 / l}\right)$.

Démonstration. On a $\operatorname{sgn}(L) \in \mathcal{L}$. Soit $Z \in \mathbf{R}$ tel que $\operatorname{deg} Z<$ $(l-1) k-1-\operatorname{deg} H$. Alors, $\operatorname{deg}(L+Z)=\operatorname{deg} L=l k$ et $\operatorname{sgn}(L+Z)=$ 
$\operatorname{sgn}(L)$, d'où le (i). De plus, d'après la définition de la fonction racine $l$-ième, $\operatorname{sgn}\left(L^{1 / l}\right)=\operatorname{sgn}\left((L+Z)^{1 / l}\right)$. D'autre part, on a

$$
Z=L+Z-L=\left((L+Z)^{1 / l}\right)^{l}-\left(L^{1 / l}\right)^{l},
$$

d'où

$$
Z=\left((L+Z)^{1 / l}-L^{1 / l}\right)\left(\sum_{j=0}^{l-1}\left((L+Z)^{1 / l}\right)^{j}\left(L^{1 / l}\right)^{l-1-j}\right) .
$$

Pour tout $j \in\{0, \ldots, l-1\}$, on a

$$
\begin{gathered}
\nu\left(\left((L+Z)^{1 / l}\right)^{j}\left(L^{1 / l}\right)^{l-1-j}\right)=-(l-1) k, \\
\operatorname{sgn}\left(\left((L+Z)^{1 / l}\right)^{j}\left(L^{1 / l}\right)^{l-1-j}\right)=\left(\operatorname{sgn}\left(L^{1 / l}\right)\right)^{l-1},
\end{gathered}
$$

d'où

$$
\nu\left(\sum_{j=0}^{l-1}\left((L+Z)^{1 / l}\right)^{j}\left(L^{1 / l}\right)^{l-1-j}\right)=-(l-1) k,
$$

et, avec (1), il vient

$$
\nu\left((L+Z)^{1 / l}-L^{1 / l}\right)=-\operatorname{deg} Z+(l-1) k \geq 2+\operatorname{deg} H,
$$

d'où

$$
\nu\left(H\left((L+Z)^{1 / l}-L^{1 / l}\right)\right) \geq 2 .
$$

On conclut avec (I.1.5).

LEMME II.1.2 (Lemme fondamental). Soit $H$ un polynôme non nul, et soit $h$ son degré. Soit $k$ un entier tel que $(l-1) k \geq h$. Alors, pour tout $\alpha \in \mathcal{L}$, tout $\zeta \in \mathbb{F}_{q}$, tout $Y \in \mathbf{R}$ de degré $<k+h$, il existe un et un seul élément $\eta=\eta(\alpha, Y)$ dans $\mathbb{F}_{q}$ tel que pour tout $Z \in \mathbf{R}$ de degré $<(l-1) k-h-1$, on ait

$$
\zeta=\operatorname{Res}\left(H\left(\alpha T^{l k}+Y T^{(l-1) k-h}+\eta T^{(l-1) k-h-1}+Z\right)^{1 / l}\right) .
$$

Démonstration. Posons $H=a_{0}+\ldots+a_{h} T^{h}$. Soient $\alpha \in \mathcal{L}, \beta=\alpha^{1 / l}$, $\zeta \in \mathbb{F}_{q}$ et $\left(y_{l k-1}, \ldots, y_{(l-1) k-h}\right) \in \mathbb{F}_{q}^{k+h}$. Si $u=\sum_{j=-\infty}^{k} u_{j} T^{j}$ est un élément de $\mathbf{K}_{\infty}$, on a

$$
\operatorname{Res}(H u)=a_{h} u_{-h-1}+a_{h-1} u_{-h}+\ldots+a_{0} u_{-1} .
$$

Pour $s=1, \ldots, k+h$, on considère la relation $\left(e_{s}\right)$ suivante :

$$
y_{l k-s}=l \beta^{l-1} u_{k-s}+\sum_{\substack{i_{1}+\ldots+i_{l}=l k-s \\ k-s<i_{1} \leq n \\ n-k<i_{1} \leq n \\ n-k<i_{l} \leq n}} u_{i_{1}} \ldots u_{i_{l}} .
$$

On détermine $u_{k-1}, \ldots, u_{-h}$ à l'aide des relations $\left(e_{1}\right), \ldots,\left(e_{k+h}\right)$. Le coefficient $a_{h}$ étant non nul, il existe un unique élément $u_{-h-1} \in \mathbb{F}_{q}$ tel que

$$
\zeta=a_{h} u_{-h-1}+a_{h-1} u_{-h}+\ldots+a_{0} u_{-1} .
$$


Il existe alors un unique élément $\eta=y_{(l-1) k-h-1} \in \mathbb{F}_{q}$ solution de l'équation $\left(e_{k+h+1}\right)$ suivante :

$$
y_{(l-1) k-h-1}=l \beta^{l-1} u_{-h-1}+\sum_{\substack{i_{1}+\ldots+i_{l}=(l-1) k-h-1 \\ k-s<i_{1} \leq n \\ n-k<i_{l} \leq n}} u_{i_{1}} \ldots u_{i_{l}} .
$$

Soit

(3) $\quad K=\alpha T^{l k}+y_{l k-1} T^{l k-1}+\ldots+y_{(l-1) k-h} T^{(l-1) k-h}+\eta T^{(l-1) k-h-1}$.

Avec (1), (2) et (I.3.2), il vient $\zeta=\operatorname{Res}\left(H K^{1 / l}\right)$, et, d'après le lemme précédent, pour tout $Z \in \mathbf{R}$ de degré $<(l-1) k-h-1$, on a $\zeta=$ $\operatorname{Res}\left(H(K+Z)^{1 / l}\right)$.

Corollaire II.1.3. Soit H un polynôme non nul. Soit $k$ un entier tel que $(l-1) k \geq \operatorname{deg} H$. Pour tout sous-ensemble $\mathbf{A} \subset \mathbf{R}$ et pour tout $i \in\{1, \ldots, r\}$, soit

$$
\sigma(\mathbf{A} ; k, i)=\sum_{\substack{A \in \mathbf{A} \\ \operatorname{deg} A=l k \\ \operatorname{sgn}(A)=\alpha_{i}}} \psi\left(\operatorname{Res}\left(H A^{1 / l}\right)\right)
$$

Alors,

(a) si $\mathbf{A}=\mathbf{L}, \sigma(\mathbf{A} ; k, i)=0$,

(b) si $\mathbf{A}=\mathbf{L} \cap \mathbf{I},|\sigma(\mathbf{A} ; k, i)| \leq(k+\operatorname{deg} H+2) q^{1+\operatorname{deg} H+k(1+l / 2)}$.

Démonstration. Posons $h=\operatorname{deg} H$. Pour $\zeta \in \mathbb{F}_{q}$, soit $\omega(\zeta)$ le nombre de $A \in \mathbf{A}$ tels que

(i) $\operatorname{deg} A=l k$,

(ii) $\operatorname{sgn}(A)=\alpha_{i}$,

(iii) $\operatorname{Res}\left(H A^{1 / l}\right)=\zeta$.

Alors,

$$
\sigma(\mathbf{A} ; k, i)=\sum_{\zeta \in \mathbb{F}_{q}} \psi(\zeta) \omega(\zeta) .
$$

Pour $Y \in \mathbf{R}$ de degré $<k+h$, soit

$$
K\left(\alpha_{i}, Y, \zeta\right)=\alpha_{i} T^{l k}+Y T^{(l-1) k-h}+\eta T^{(l-1) k-h-1},
$$

où $\eta=\eta\left(\alpha_{i}, Y\right)$ est l'élément défini au lemme fondamental tel que

$$
\operatorname{Res}\left(H K\left(\alpha_{i}, Y, \zeta\right)^{1 / l}\right)=\zeta .
$$

Soit aussi $a(l k ; Y, \zeta)$ le nombre de $A \in \mathbf{A}$ tels que

$$
\operatorname{deg}\left(A-K\left(\alpha_{i}, Y, \zeta\right)\right)<(l-1) k-h-1 .
$$


Alors

$$
\omega(\zeta)=\sum_{\substack{Y \in \mathbf{R} \\ \operatorname{deg} Y<k+h}} a(l k ; Y, \zeta) .
$$

Si $\mathbf{A}=\mathbf{L}, a(l k ; Y, \zeta)=q^{(l-1) k-h-1}$, et

$$
\sigma(\mathbf{A} ; k, i)=q^{l k-1} \sum_{\zeta \in \mathbb{F}_{q}} \psi(\zeta) .
$$

Le caractère $\psi$ étant non principal, $\sigma(\mathbf{A} ; k, i)=0$.

On suppose $\mathbf{A}=\mathbf{L} \cap \mathbf{I}$. Alors $a(l k ; Y, \zeta)$ est le nombre de polynômes irréductibles $P$ tels que

$$
\operatorname{deg}\left(P-K\left(\alpha_{i}, Y, \zeta\right)\right)<(l-1) k-h-1 .
$$

D'après [8], théorème 4 ,

$$
a(l k ; Y, \zeta)=\frac{q^{(l-1) k-h-1}}{l k}+e(l k ; Y, \zeta),
$$

avec

$$
|e(l k ; Y, \zeta)| \leq(k+h+2) q^{l k / 2}
$$

d'où

$$
\begin{gathered}
\sigma(\mathbf{A} ; k, i)=\frac{q^{l k-1}}{l k} \sum_{\zeta \in \mathbb{F}_{q}} \psi(\zeta)+\sum_{\zeta \in \mathbb{F}_{q}} \psi(\zeta) \sum_{\substack{Y \in Z \\
\operatorname{deg} Y<k+h}} e(l k ; Y, \zeta), \\
|\sigma(\mathbf{A} ; k, i)| \leq q^{k+h+1}(k+h+2) q^{l k / 2} .
\end{gathered}
$$

II.2. Démonstration du théorème. On désigne par A l'un de deux ensembles $\mathbf{L}$ ou $\mathbf{L} \cap \mathbf{I},\left(A_{n}\right)$ désigne la suite $\left(L_{n}\right)$ si $\mathbf{A}=\mathbf{L}$, la suite $\left(P_{n}\right)$ si $\mathbf{A}=\mathbf{L} \cap \mathbf{I}$. Dans $\mathbf{R}$, il y a $q^{m}$ polynômes unitaires de degré $m$. Soit $\pi_{m}$ le nombre de polynômes irréductibles unitaires de R. On a (cf. [6], p. 82)

$$
q^{m}-2 q^{m / 2} \leq m \pi_{m} \leq q^{m} .
$$

Dans $\mathbf{A}$, il y a donc $a_{m}$ polynômes de degré $l m$, avec

$$
a_{m}= \begin{cases}r q^{l m} & \text { si } \mathbf{A}=\mathbf{L} \\ r \pi_{l m} & \text { si } \mathbf{A}=\mathbf{L} \cap \mathbf{I}\end{cases}
$$

et il y a $b_{s}$ polynômes de degré $\leq s$, avec

$$
b_{s}=a_{1}+\ldots+a_{s} .
$$

La suite $\left(b_{s}\right)$ est strictement croissante. De plus,

$$
b_{s}=\frac{r\left(q^{l(s+1)}-q^{l}\right)}{q^{l}-1} \quad \text { si } \mathbf{A}=\mathbf{L},
$$


et il existe deux constantes strictement positives $\kappa_{1}=\kappa_{1}(q, l)$ et $\kappa_{2}=\kappa_{2}(q, l)$ telles que

$$
\kappa_{1} \frac{q^{l s}}{s} \leq b_{s} \leq \kappa_{2} \frac{q^{l s}}{s} \quad \text { si } \mathbf{A}=\mathbf{L} \cap \mathbf{I} .
$$

Soit $H \in \mathbf{R}^{*}$. Soit

$$
h=\operatorname{deg} H .
$$

Soit $N$ un entier assez grand pour que les conditions suivantes soient satisfaites :

$$
N>b_{\lceil 1+(h+1) /(l-1)\rceil}, \quad N>N_{h, l},
$$

$\lceil x\rceil$ désignant le plus petit entier $\geq x, N_{h, l}$ étant égal à 0 , si $\mathbf{A}=\mathbf{L}$, et tel que l'implication suivante soit vraie :

$$
\kappa_{2} \frac{q^{l t}}{l t} \geq N_{h, l} \Rightarrow \frac{q^{(l / 2-1) t}}{t^{2}} \geq 4 l q^{h} \quad \text { et } \quad t \geq h+2
$$

si $\mathbf{A}=\mathbf{L} \cap \mathbf{I}$. Notons que cette implication ne peut avoir lieu que si $l \geq 3$.

Dans le cas où $\mathbf{A}=\mathbf{L} \cap \mathbf{I}$, on supposera donc $l \geq 3$.

Soit

$$
W(N)=\sum_{n=1}^{N} E\left(H\left(A_{n}\right)^{1 / l}\right) .
$$

Dans ce qui suit, une somme portant sur l'ensemble vide sera supposée nulle.

Il existe un et un seul entier $t$ tel que

$$
b_{t-1} \leq N<b_{t} .
$$

On pose

$$
S_{1}=\sum_{n=1}^{b_{t-1}} E\left(H\left(A_{n}\right)^{1 / l}\right) .
$$

Si $N>b_{t-1}$, il existe un unique entier $s \in\{0, \ldots, r-1\}$ tel que

$$
1+b_{t-1}+s a_{t} \leq N \leq b_{t-1}+(s+1) a_{t} .
$$

On pose

$$
\begin{aligned}
& S_{2}=\sum_{j=0}^{s-1} \sum_{n=1+b_{t-1}+j a_{t}}^{b_{t-1}+(j+1) a_{t}} E\left(H\left(A_{n}\right)^{1 / l}\right) \quad \text { si } N<b_{t-1}+(s+1) a_{t}, \\
& S_{2}=\sum_{j=0}^{s} \sum_{n=1+b_{t-1}+j a_{t}}^{b_{t-1}+(j+1) a_{t}} E\left(H\left(A_{n}\right)^{1 / l}\right) \quad \text { si } N=b_{t-1}+(s+1) a_{t} .
\end{aligned}
$$


Enfin, si $N<b_{t-1}+(s+1) a_{t}$, on pose

$$
S_{3}=\sum_{n=1+b_{t-1}+s a_{t}}^{N} E\left(H\left(A_{n}\right)^{1 / l}\right) .
$$

Trivialement, on a

$$
W(N)=S_{1}+S_{2}+S_{3}
$$

Proposition II.2.1. (a) $S i \mathbf{A}=\mathbf{L}$, on a

$$
S_{2}=0, \quad\left|S_{1}\right| \leq q^{1+h /(l-1)} .
$$

(b) Si $\mathbf{A}=\mathbf{L} \cap \mathbf{I}$, on a

$$
\left|S_{1}\right| \leq r(t+h+1) q^{h+t(1+l / 2)}+q^{1+h /(l-1)}, \quad\left|S_{2}\right| \leq r(t+h+2) q^{1+h+t(1+l / 2)} .
$$

Démonstration. La somme $S_{1}$ porte sur les polynômes $A \in \mathbf{A}$ de degré $\leq l(t-1)$, la somme $S_{2}$, lorsqu'elle n'est pas vide, porte soit sur les polynômes $A \in \mathbf{A}$ de degré $l t$ tels que $\operatorname{sgn}(A) \in\left\{\alpha_{1}, \ldots, \alpha_{s-1}\right\}$ si $N<$ $b_{t-1}+(s+1) a_{t}$, soit sur les polynômes $A \in \mathbf{A}$ de degré $l t$ tels que $\operatorname{sgn}(A) \in$ $\left\{\alpha_{1}, \ldots, \alpha_{s}\right\}$ si $N=b_{t-1}+(s+1) a_{t}$. Donc

$$
S_{1}=\sum_{\substack{A \in \mathbf{A} \\
(l-1) \operatorname{deg} A \leq h}} E\left(H A^{1 / l}\right)+\sum_{\substack{k \leq t-1 \\
(l-1) k>h}} \sum_{i=1}^{r} \sum_{\begin{array}{c}
A \in \mathbf{A} \\
\operatorname{deg} A=l k \\
\operatorname{sgn}(A)=\alpha_{i}
\end{array}} E\left(H A^{1 / l}\right) .
$$

La première somme est majorée par $q^{1+h /(l-1)}$. D'après le corollaire au lemme fondamental, si $\mathbf{A}=\mathbf{L}$, la deuxième somme vaut 0 et, si $\mathbf{A}=\mathbf{L} \cap \mathbf{I}$, la deuxième somme est majorée par

$$
\sum_{\substack{k \leq t-1 \\(l-1) k>h}} \sum_{i=1}^{r}(k+h+2) q^{1+h+k+k l / 2} \leq r(t+h+1) q^{h+t(1+l / 2)} .
$$

La somme $S_{2}$ se traite de même.

Proposition II.2.2. (a) Si $\mathbf{A}=\mathbf{L}$, on a

$$
\left|S_{3}\right| \leq q^{(l-1) t-h} \text {. }
$$

(b) $S i \mathbf{A}=\mathbf{L} \cap \mathbf{I}$, on a

$$
\left|S_{3}\right| \leq 2 q^{(l-1) t-h}+(t+h+2) q^{t+t l / 2+h} .
$$

Démonstration. Nous supposerons $N<b_{t-1}+(s+1) a_{t}$, car, sinon, il n'y a rien à démontrer. Posons

$$
j=1+s a_{t}+b_{t-1} .
$$


Alors, avec (II.2.15),

$$
S_{3}=\sum_{n=j}^{N} E\left(H\left(A_{n}\right)^{1 / l}\right)
$$

d'où

$$
\left|S_{3}\right| \leq N-j+1
$$

Les polynômes $A_{i}$ intervenant dans $S_{3}$ s'écrivent

$$
A_{i}=\alpha_{i} T^{l t}+H_{n_{i}}, \quad j \leq i \leq N,
$$

la suite $\left(H_{n_{i}}\right)$ étant strictement croissante dans $\mathbf{R}$. On écrit $n_{N}$ en base $q$ :

$$
n_{N}=c_{0}+c_{1} q^{t}+\ldots+c_{m} q^{m} .
$$

On distingue deux cas.

Premier cas : $m \leq(l-1) t-h-1$. La suite $\left(H_{n}\right)$ étant indexée suivant l'ordre croissant, la suite $\left(n_{i}\right)$ est aussi strictement croissante, d'où

$$
N-j \leq n_{N}-n_{j} \leq n_{N} \leq q^{m+1}-1,
$$

et, avec (2),

$$
\left|S_{3}\right| \leq q^{(l-1) t-h} .
$$

Deuxième cas: $m>(l-1) t-h-1$. On a

$$
H_{n_{N}}=\mathcal{X}_{c_{0}}+\mathcal{X}_{c_{1}} T+\ldots+\mathcal{X}_{c_{m}} T^{m} .
$$

Les polynômes $H_{n_{i}}$ intervenant dans la somme $S_{3}$ sont de la forme

$$
H_{n_{i}}=y_{0}+y_{1} T+\ldots+y_{m} T^{m} \leq \mathcal{X}_{c_{0}}+\mathcal{X}_{c_{1}} T+\ldots+\mathcal{X}_{c_{m}} T^{m} .
$$

Soit $\mathcal{Y}$ l'ensemble des polynômes

$$
Y=y_{(l-1) t-h}+y_{(l-1) t-h+1} T+\ldots+y_{m} T^{m-(l-1) t+h}
$$

tels que pour tout polynôme $W \in \mathbf{R}$ de degré $<(l-1) t-h$, on ait

$$
Y T^{(l-1) t-h}+W \leq H_{n_{N}} .
$$

Soit $\mu$ le plus grand des indices $i \in\{j, \ldots, N\}$ pour lesquels $A_{i}$ s'écrive

$$
A_{i}=\alpha_{s} T^{l t}+Y T^{(l-1) t-h}+W,
$$

avec $Y \in \mathcal{Y}$ et $W \in \mathbf{R}$ vérifiant $\operatorname{deg} W<(l-1) t-h$. On pose alors

$$
\begin{aligned}
& S_{4}=\sum_{i=j}^{\mu} E\left(H\left(A_{i}\right)^{1 / l}\right), \\
& S_{5}=\sum_{i=\mu+1}^{N} E\left(H\left(A_{i}\right)^{1 / l}\right) .
\end{aligned}
$$


On a

$$
S_{3}=S_{4}+S_{5}
$$

On procède comme pour le corollaire au lemme fondamental. On a

$$
S_{4}=\sum_{\zeta \in \mathbb{F}_{q}} \psi(\zeta) \omega(\zeta),
$$

où, pour $\zeta \in \mathbb{F}_{q}, \omega(\zeta)$ désigne le nombre de couples $(Y, W)$ tels que

(i) $Y \in \mathcal{Y}$,

(ii) $W \in \mathbf{R}, \operatorname{deg} W<(l-1) t-h$,

(iii) $\left(\alpha_{s} T^{l t}+Y T^{(l-1) t-h}+W\right) \in \mathbf{A}$,

(iv) $\operatorname{Res}\left(H\left(\alpha T^{l t}+Y T^{(l-1) t-h}+W\right)^{1 / l}\right)=\zeta$.

Ici encore

$$
\omega(\zeta)=\sum_{Y \in \mathcal{Y}} a(l t ; Y, \zeta),
$$

où $a(l t ; Y, \zeta)$ désigne toujours le nombre de $A \in \mathbf{A}$ tels que

$$
\operatorname{deg}\left(A-K\left(\alpha_{i}, Y, \zeta\right)\right)<(l-1) t-h-1
$$

avec

$$
K\left(\alpha_{i}, Y, \zeta\right)=\alpha_{i} T^{l t}+Y T^{(l-1) t-h}+\eta\left(\alpha_{i}, Y\right) T^{(l-1) t-h-1},
$$

$\eta\left(\alpha_{i}, Y\right)$ étant l'élément défini au lemme fondamental. On obtient

$$
S_{4}=0 \quad \text { si } \mathbf{A}=\mathbf{L} .
$$

On suppose $\mathbf{A}=\mathbf{L} \cap \mathbf{I}$. Alors,

$$
\left|S_{4}\right| \leq \#(\mathcal{Y})(t+h+2) q^{t l / 2},
$$

d'où

$$
\left|S_{4}\right| \leq(t+h+2) q^{t(l / 2+1)+h} \quad \text { si } \mathbf{A}=\mathbf{L} \cap \mathbf{I} .
$$

Trivialement, si $S_{5}$ n'est pas la somme vide,

$$
\left|S_{5}\right| \leq N-\mu \text {. }
$$

Dans l'écriture de $n_{N}$ en base $q$, soit $\tau$ le plus petit indice $>(l-1) t-h$ tel que $c_{\tau} \neq 0$. En fait,

$$
n_{N}=c_{m} q^{m}+c_{m-1} q^{m-1}+\ldots+c_{\tau} q^{\tau}+c_{(l-1) t-h-1} q^{(l-1) t-h-1}+\ldots+c_{0} .
$$

Tout polynôme

$$
A=\alpha_{s} T^{l t}+y_{m} T^{m}+y_{m-1} T^{m-1}+\ldots+y_{\tau} q^{\tau}+y_{\tau-1} q^{\tau-1}+\ldots+y_{0},
$$

dont les coefficients $\left(y_{i}\right)$ vérifient les conditions $(\mathcal{C})$ suivantes :

(C) $y_{m} \leq \mathcal{X}_{c_{m}}, \quad y_{m-1} \leq \mathcal{X}_{c_{m-1}}, \ldots, y_{\tau+1} \leq \mathcal{X}_{c_{\tau+1}}, \quad y_{\tau}<\mathcal{X}_{c_{\tau}}$, 
appartient à l'ensemble $\mathbf{L}$ et est inférieur à $A_{n_{N}}$. Dans le cas où $\mathbf{A}=\mathbf{L}$, on a donc

$$
\begin{gathered}
n_{\mu} \geq c_{m} q^{m}+c_{m-1} q^{m-1}+\ldots+\left(c_{\tau}-1\right) q^{\tau}+(q-1) q^{\tau-1}+\ldots+(q-1), \\
n_{\mu} \geq n_{N}-q^{(l-1) t-h} .
\end{gathered}
$$

Supposons $\mathbf{A}=\mathbf{L} \cap \mathbf{I}$. D'après [8], théorème 4, pour tout polynôme

$$
Y=\alpha_{s} T^{l t}+y_{m} T^{m}+y_{m-1} T^{m-1}+\ldots+y_{\tau} q^{\tau}+\ldots+y_{(l-1) t-h} T^{(l-1) t-h}
$$

dont les coefficients $\left(y_{i}\right)$ vérifient les conditions $(\mathcal{C})$, il existe

$$
\Pi(l t ; Y,(l-1) t-h)=\frac{q^{(l-1) t-h}}{l t}+\varrho(l t ; Y,(l-1) t-h)
$$

polynômes irréductibles $P \in \mathbf{R}$ tels que $\operatorname{deg}(P-Y)<(l-1) t-h$ avec

$$
|\varrho(l k ; Y,(l-1) t-h)| \leq(t+h+2) q^{l t / 2} .
$$

De tels polynômes $P$ appartiennent à $\mathbf{L} \cap \mathbf{I}=\mathbf{A}$. On a supposé $N>N_{l, h}$. On a donc

$$
\begin{gathered}
\frac{q^{(l-1) t-h}}{l t} \geq 4 t q^{l t / 2} \geq 2(t+h+2) q^{l t / 2}, \\
\Pi(l t ; Y,(l-1) t-h) \geq q^{(l-1) t-h} /(2 l t) \geq 1 .
\end{gathered}
$$

Ici,

$$
\begin{gathered}
n_{\mu} \geq c_{m} q^{m}+c_{m-1} q^{m-1}+\ldots+\left(c_{\tau}-1\right) q^{\tau}+(q-1) q^{\tau-1}+\ldots+(q-1) q^{(l-1) t-h}, \\
n_{\mu} \geq n_{N}-2 q^{(l-1) t-h}+1 .
\end{gathered}
$$

D'où

$$
N-\mu \leq \begin{cases}q^{(l-1) t-h} & \text { si } \mathbf{A}=\mathbf{L}, \\ 2 q^{(l-1) t-h}-1 & \text { si } \mathbf{A}=\mathbf{L} \cap \mathbf{I} .\end{cases}
$$

On conclut avec (4), (7)-(10).

Proposition II.2.3. On a

$$
\begin{aligned}
&\left|\frac{1}{N} \sum_{n=1}^{N} E\left(H\left(A_{n}\right)^{1 / l}\right)\right| \ll N^{-1 / l} \quad \text { si } \mathbf{A}=\mathbf{L}, \\
&\left|\frac{1}{N} \sum_{n=1}^{N} E\left(H\left(A_{n}\right)^{1 / l}\right)\right| \ll(\log N)^{3 / 2+1 / l} N^{1 / l-1 / 2} \\
& \text { si } \mathbf{A}=\mathbf{L} \cap \mathbf{I} \text { et } \text { si } 3 \leq l \leq 4,
\end{aligned}
$$

$$
\left|\frac{1}{N} \sum_{n=1}^{N} E\left(H\left(A_{n}\right)^{1 / l}\right)\right| \ll(\log N)^{1-1 / l} N^{-1 / l}
$$

si $\mathbf{A}=\mathbf{L} \cap \mathbf{I}$ et si $l>5$,

les constantes impliquées par le symbole «ne dépendant que de q et de l. 
Démonstration. On se place d'abord dans le cas $\mathbf{A}=\mathbf{L}$. Avec (II.2.9), (II.2.16), les propositions II.2.1 et II.2.2, il vient

$$
\left|\frac{1}{N} \sum_{n=1}^{N} E\left(H\left(A_{n}\right)^{1 / l}\right)\right| \leq \frac{1}{N}\left(q^{(l-1) t-h}+q^{1+h /(l-1)}\right),
$$

puis, avec (II.2.10) et (II.2.4), on a

$$
\begin{gathered}
\left|\frac{1}{N} \sum_{n=1}^{N} E\left(H\left(A_{n}\right)^{1 / l}\right)\right| \leq \frac{\left(q^{(l-1) t-h}+q^{1+h /(l-1)}\right)\left(q^{l}-1\right)}{r\left(q^{l t}-q^{l}\right)}, \\
\left|\frac{1}{N} \sum_{n=1}^{N} E\left(H\left(A_{n}\right)^{1 / l}\right)\right| \ll q^{-t} \ll N^{-1 / l}
\end{gathered}
$$

d'où (II.2.17).

On se place maintenant dans le cas $\mathbf{A}=\mathbf{L} \cap \mathbf{I}$. Il vient alors

$$
\begin{aligned}
& \left|\frac{1}{N} \sum_{n=1}^{N} E\left(H\left(A_{n}\right)^{1 / l}\right)\right| \\
& \quad \leq \frac{1}{N}\left(q^{1+h /(l-1)}+(2 r+1)(t+h+2) q^{1+h+t(1+l / 2)}+2 q^{(l-1) t-h}\right) .
\end{aligned}
$$

Les relations (II.2.10) et (II.2.5) donnent alors

$$
\left|\frac{1}{N} \sum_{n=1}^{N} E\left(H\left(A_{n}\right)^{1 / l}\right)\right| \ll \frac{t^{2} q^{t(1+l / 2)}+t q^{(l-1) t}}{q^{l t}} .
$$

Pour $l \leq 4$,

$$
\begin{gathered}
\left|\frac{1}{N} \sum_{n=1}^{N} E\left(H\left(A_{n}\right)^{1 / l}\right)\right| \ll t^{2} q^{t(1-l / 2)}, \\
\left|\frac{1}{N} \sum_{n=1}^{N} E\left(H\left(A_{n}\right)^{1 / l}\right)\right| \ll(\log N)^{3 / 2+1 / l} N^{1 / l-1 / 2},
\end{gathered}
$$

d'où (II.2.18). Pour $l>4$,

$$
\left|\frac{1}{N} \sum_{n=1}^{N} E\left(H\left(A_{n}\right)^{1 / l}\right)\right| \ll t q^{-t} \ll(\log N)^{1-1 / l} N^{-1 / l},
$$

d'où (II.2.19).

Le critère de Weyl, théorème I.2.2, nous donne alors le résultat annoncé.

Dans le cas $\mathbf{A}=\mathbf{L} \cap \mathbf{I}$, la restriction $l \geq 3$ provient du fait suivant. Dans la démonstration intervient en fait le nombre $I\left(l k ; y_{l k}, y_{(l-1) k-1}, \ldots\right.$, $\left.y_{(l-1) k-h-1}\right)$ de polynômes irréductibles $P$ de degré $l k$, 


$$
\begin{aligned}
P=y_{l k} T^{l k}+y_{l k-1} T^{l k-1}+\ldots+y_{(l-1) k-1} T^{(l-1) k-1}+\ldots & \\
& \ldots+y_{(l-1) k-h-1} T^{(l-1) k-h}+\ldots+y_{0},
\end{aligned}
$$

dont les coefficients $y_{l k}, y_{(l-1) k-1}, \ldots, y_{(l-1) k-h-1}$ sont fixés. Aucune approximation de ce nombre n'étant connue, on utilise un théorème de répartition des polynômes irréductibles qui donne le nombre $\Pi(l k, Y, l k-h-1)$ de polynômes irréductibles $P$ de degré $l k$, de la forme

$$
P=Y+y_{(l-1) k-h-2} T^{(l-1) k-h-2}+\ldots+y_{0},
$$

dont les coefficients $y_{l k}, y_{l k-1}, \ldots, y_{(l-1) k-1}, \ldots, y_{(l-1) k-h-1}$ sont fixés. Ceci introduit un terme d'erreur d'ordre $k q^{k+l k / 2}$ plus grand que l'ordre du terme principal lorsque $l=2$.

On pourrait penser établir pour le nombre $I\left(l k ; y_{l k}, y_{(l-1) k-1}, \ldots\right.$ $\left.\ldots, y_{(l-1) k-h-1}\right)$ un théorème analogue aux théorèmes établis dans [8] ou [5] pour les nombres $\Pi(l k ; Y, l k-h-1)$. Mais cela semble être un problème très difficile.

\section{Bibliographie}

[1] L. Carlitz, Diophantine approximations in fields of characteristic p, Proc. Amer. Math. Soc. 3 (1952), 187-208.

[2] A. Dijksma, Uniform distribution of polynomials over $G F\{q, x\}$ in $G F[q, x]$, part I, Nederl. Akad. Wetensch. Proc. Ser. A 72 (1969), 376-383.

[3] - Uniform distribution of polynomials over $G F\{q, x\}$ in $G F[q, x]$, part II, ibid. 73 (1970), 187-195.

[4] D. R. Hayes, The expression of a polynomial as a sum of three irreducibles, Acta Arith. 11 (1966), 461-488.

[5] - The distribution of irreducibles in $G F[q, x]$, Trans. Amer. Math. Soc. 117 (1965), 101-127.

[6] R. Lidl and H. Niederreiter, Introduction to Finite Fields and Their Applications, Cambridge University Press, 1986.

[7] D. de Mathan, Approximations diophantiennes dans un corps local, Bull. Soc. Math. France Mém. 21 (1970).

[8] G. Rhin, Répartition modulo 1 dans un corps de séries formelles sur un corps fini, Dissertationes Math. 95 (1972).

LABORATOIRE DE MATHÉMATIQUES

CASE 322

FACULTÉ DES SCIENCES DE SAINT-JÉRÔME

AVENUE ESCADRILLE NORMANDIE-NIEMEN

13397 MARSEILLE CEDEX 20, FRANCE 